\title{
New parameter for defining a square: Exact solution to squaring the circle; proving $\pi$ is rational
}

\author{
Lorna A. Willis

\section{Email address:} \\ lana latetia@yahoo.com, lana.latetia@gmail.com
}

Department of Physics, Faculty of Science and Technology, University of the West Indies, Mona Campus, Jamaica, West Indies

\section{To cite this article:}

Lorna A. Willis. New Parameter for Defining a Square: Exact Solution to Squaring the Circle; Proving $\pi$ is Rational. American Journal of Applied Mathematics. Vol. 2, No. 3, 2014, pp. 74-78. doi: 10.11648/j.ajam.20140203.11

\begin{abstract}
Historically, mathematicians sought for a unique relationship between a square and a circle of equal area without much success. The ratio of perimeter of a circle to its diameter is known and given as the symbol $\pi$. However, $\pi$ was deemed IRRATIONAL. By using the concept of a TESSELLATION, that is the tiling of a plane using one or more geometric shapes, called tiles, with no overlaps and no gaps, a square is described for the first time, as an equi-edge juxtaposition of eight identical right isosceles triangles. The usual median of a triangle is consistently identified in each of these triangles and is designated SECONDARY MEDIAN in relation to a square. There are eight Secondary Medians in a square. When the size of the Secondary Median of a square matches the size of the radius of a circle, and the two shapes are placed so that their centers are coincident, it is established that the areas of the two shapes are equal, thereby demonstrating the basis for the exact solution to the ancient geometric construction problem- SQUARING THE CIRCLE, with the consequences that; 1) $\pi$ is, unambiguously a feature of the area of a square, 2) $\pi$ is rational, has an exact value of 3.2 , from any circle, a square of equal area is constructed in finite steps as well as the converse, 3) a square and an ellipse of equal area can be constructed, 4) $\pi$ is not a feature limited to circles and associated shapes, as has been historically documented, but is a feature of Euclidian Geometry. Exact value of $\pi$ means formulae featuring $\pi$ are unchanged qualitatively, but changes slightly, quantitatively.
\end{abstract}

Keywords: Tessellation, Secondary Median, Exact

\section{Introduction}

Geometry is about relationship, that is connectedness, logical reasoning. It involves points, lines, angles, plane shapes, and their interrelationships. Geometric construction demonstrates, visually, these relationships by the use of only a straight edge and a compass.

In general, it is about proceeding from what is known to determining what needs to be known.

Any shape can be constructed on a plane. Groups of shapes conforming to like features are given a specific name e.g. quadrilateral i.e. having four edges; triangle i.e. having three edges; circle i.e. having one edge.

Historically, mathematicians sought for a unique relationship between a square and a circle and more generally, between a polygon, i.e. many edges, and a circle. Finding a relationship between a square and a circle was evidently, inherently difficult, since the square has four equal edges forming four right angles, and the circle has one edge that is a closed, curved line.
From a visual perspective, a square has a centre and so does a circle. By describing a square, in a new and profoundly creative way, namely, as an equi-edge tessellation of eight identical isosceles triangles, a new parameter, designated the secondary median, s, is revealed as shown later. This parameter is used to provide an alternative definition of a square and by so doing, a second and critical relationship between the square and a circle is established.

\section{Definitions, Theorems and Proofs}

Historically, the ratio of perimeter of a circle to diameter, named pi and given the symbol $\pi$, was known to the Ancient Egyptians (c 2000 BC). By careful observation and measurement, mathematicians historically, have demonstrated that $\pi$ is a feature of a circle. They DID NOT associate $\pi$ with any other shape. With an established basis for relating a square to a circle it is demonstrable that $\pi$ is also a feature of a square and indeed of any other shape.

By using visual, creative, and critical thinking, a square is 
described and defined in a manner not previously done. The main idea influencing this approach is the forging of the revelation of a new and meaningful parameter that would have two purposes, viz.

- $\quad$ Provide a basis for defining a square; and

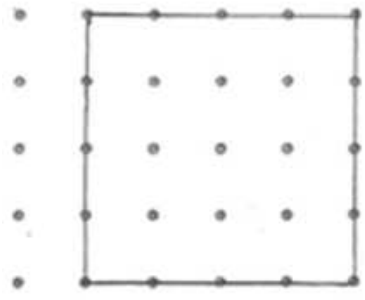

Square

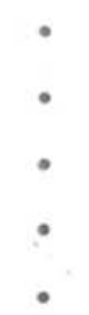

\section{Tessellation}

- Establish a correlation with the parameter that defines a circle

The diagrams below demonstrate the realization of these objectives

Figure 1. There are three figures illustrated here

This parameter allows for matching exactly any square to a circle of equal area as shown

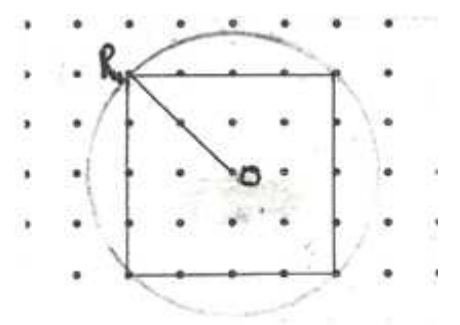

a)

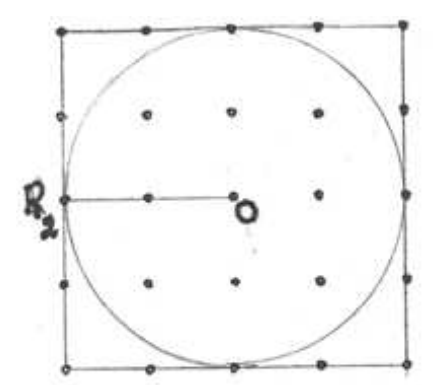

b)

Figure 2. There are two figures illustrated here

Using point 0 , and the line $\mathrm{R}_{2} \mathrm{R}_{0} \mathrm{R}_{1}$ as coherent references, point $\mathrm{R}_{1}$ defines the parameter $\mathrm{R}_{1} \mathrm{O}$ that identifies the radius of the circle of maximum area associated with the square.

Point $\mathrm{R}_{2}$, defines the parameter $\mathrm{R}_{2} \mathrm{O}$ that identifies the radius of the circle of minimum area associated with the same square.

Line $R_{2} R_{0} R_{1}$ is associated with a linear scale, and point $R 0$, being midway between $R_{1}$ and $R_{2}$, a maximum and a minimum, is a neutral point or zero.

Hence, $\mathrm{R}_{0}$ defines the parameter $\mathrm{R}_{0} \mathrm{O}$, which defines the radius of the circle which has an area equal to the area of the referred square.

This demonstration leads to the General Theorem designated the Willis-Lawrence theorem which states that;

If the secondary median of a square and the radius of a circle have the same magnitude then the square and the circle have the same area.

Additionally, this demonstration is a presentation of the exact solution to the famous ancient geometric construction problem referred to as- squaring the circle, which means-"...finding a length e for the edge of the square so that $\mathrm{e}^{2}=\pi \mathrm{r}^{2}$, where $\mathrm{r}$ is the radius of the circle. This means $\mathrm{e}$ $=\mathrm{r} \sqrt{ } \pi$."

The geometric constructions that are accomplished from the Willis-Lawrence theorem are as follows:

1) Construction from a square, this square and circle of equal area using a straight edge and compass only. *

Construct the square

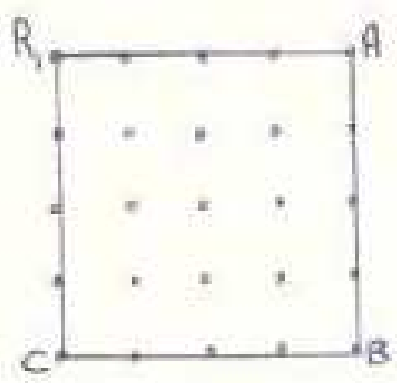

Figure 3

Connect opposite vertices $\mathrm{R}_{1}$ and $\mathrm{B}$ and $\mathrm{A}$ and $\mathrm{C}$ to obtain diagonals and identify centre, $\mathrm{O}$

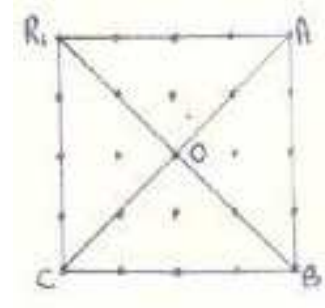

Figure 4

Use two bisecting operations of $\mathrm{R}_{1} \mathrm{C}$ to identify point $\mathrm{R}_{0}$ 


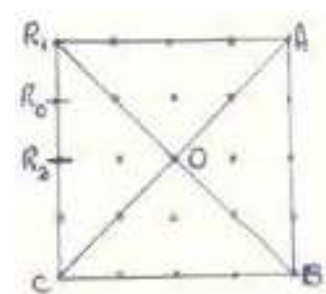

Figure 5

Connect points $\mathrm{R}_{0}$ and $\mathrm{O}$ to determine a secondary median, $\mathrm{OR}_{0}$

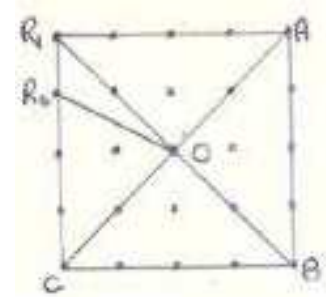

Figure 6

Using compass extent of size $\mathrm{OR}_{0}$ construct the circle

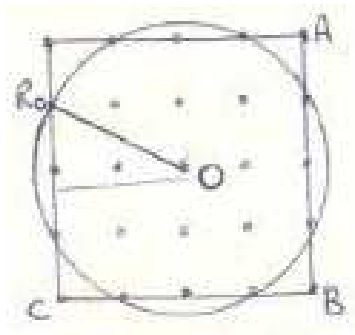

Figure 7

2) Construction from circle, this circle and a square of equal area

Construct a right triangle, in which the legs are in the ratio $1: 2$

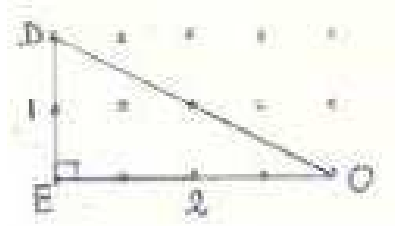

Figure 8

Using compass extent equal to the radius of the referred circle, identify, along $\mathrm{OD}$, this radius $\mathrm{OR}_{0}$

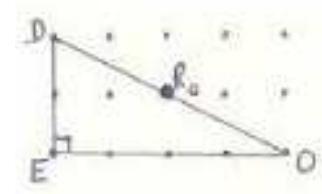

Figure 9

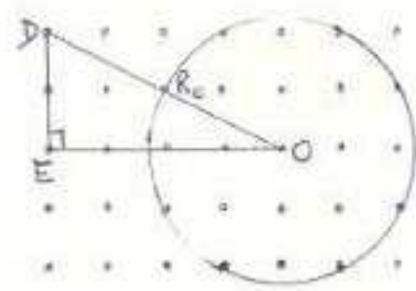

Figure 10

Construct a perpendicular $\mathrm{R}_{0} \mathrm{~F}$ from $\mathrm{R}_{0}$ to $\mathrm{OE}$

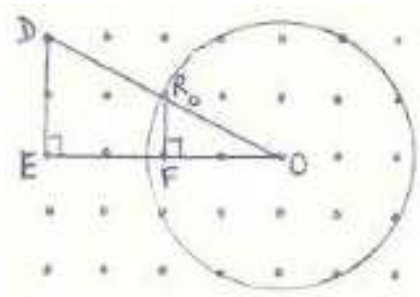

Figure 11

With compass extent equal to twice $\mathrm{OF}$, that is the edge e of the square, complete the construction of the square.

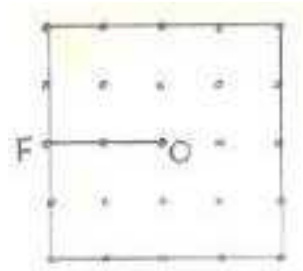

Figure 12

The foregoing constructions are accomplished using one parameter with two different names, viz. secondary median and radius, of the same size, refuting history's claim- "In 1882 , it was finally proved that it was impossible by any geometric construction."

The dotted grid is associated with linear scales in both the $\mathrm{x}$ and $\mathrm{y}$ directions. An arbitrary unit of 1 (one) is assigned to the interval between consecutive dots in both directions. Using this information, the area of the circle defined by radius $\mathrm{R}_{0} \mathrm{O}$, is $\pi\left(\mathrm{R}_{0} \mathrm{O}\right)^{2}$ or $\pi \mathrm{r}^{2}$, where $\mathrm{r}_{0}$ is the symbol for its radius.

Applying the Pythagorean Theorem to triangle $\mathrm{OR}_{2} \mathrm{R}_{0}$, leads to

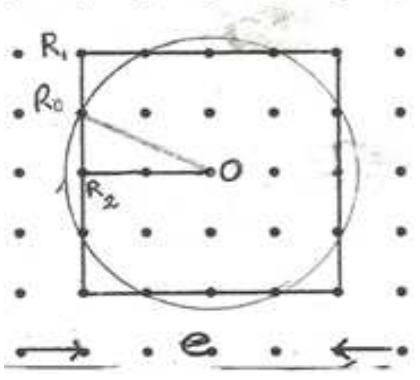

Figure 13

Construct the circle 
$\left(\mathrm{OR}_{0}\right)^{2}=\left(\mathrm{R}_{0} \mathrm{R}_{2}\right)^{2}+\left(\mathrm{R}_{2} \mathrm{O}\right)^{2}$

i.e. $\left(\mathrm{OR}_{0}\right)^{2}=(1)^{2}+(2)^{2}$

or $\left(\mathrm{OR}_{0}\right)^{2}=5$

So, $r^{2}=5$

So, the area of this circle is $5 \pi$, and the area of the square must be $5 \pi$, also. However, the area of the square is (its edge $)^{2}$, i.e. $(4)^{2}=16$

Hence, $5 \pi=16$

Or $\pi=16 / 5$

i.e. $\pi=3.2$ this is rational

With reference to history's requirement that e $=r \sqrt{ } \pi$

The willis-lawrence demonstration shows

$\mathrm{e}=4, \mathrm{r}=\sqrt{ } 5 ; \pi=16 / 5$, so

$4=\sqrt{5} \sqrt{ } 16 / 5$

Or $4=4$ a compliant result which is $e=r \sqrt{ }$

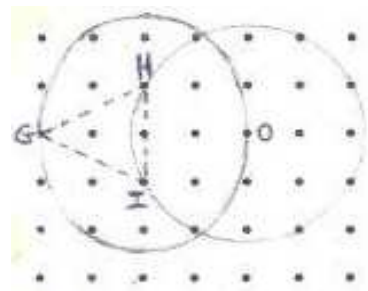

In the diagram $\mathrm{OH}$ and $\mathrm{OI}$ are radii of the circle of radius, r. Points $\mathrm{H}$ and $\mathrm{I}$ are the foci of the ellipse and $\mathrm{GH}=\mathrm{GI}=\mathrm{OH}=\mathrm{OI}=\mathrm{r}$

Area of circle $=\pi(\mathrm{OH})^{2}=\pi \mathrm{r}^{2}$

Area of the ellipse $=\pi(\mathrm{GI})(\mathrm{GH})=\pi \mathrm{r}^{2}$

Hence, circle and ellipse are of equal area.

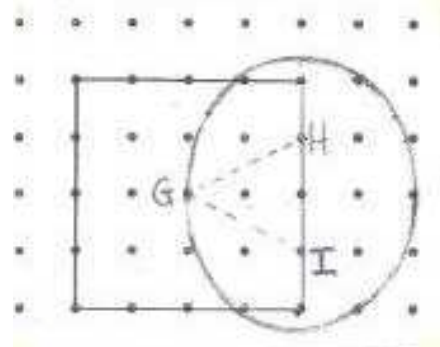

With reference to the diagram, it has been established that the area of the square is $\pi r^{2}$. So the area of square and ellipse are equal. So the inference is that $\pi$ is a feature of the area of a circle, a square and an ellipse.

Figure 14

The diagrams that follow including the circle and the ellipse, demonstrate that a fixed area can be bound by different outlines or shapes. Any square can be conceptualized as a tessellation of many squares of any size. Each of these can be transformed iteratively into other shapes or composite of shapes of the same area, using the techniques of division, dissection, construction from one shape to another, permutation and combination and varying orientations. So that one shape can evolve into an endless array of different shapes of the same area, highlighting $\pi$ as a feature of each shape, and indeed a feature of Euclidean Geometry.

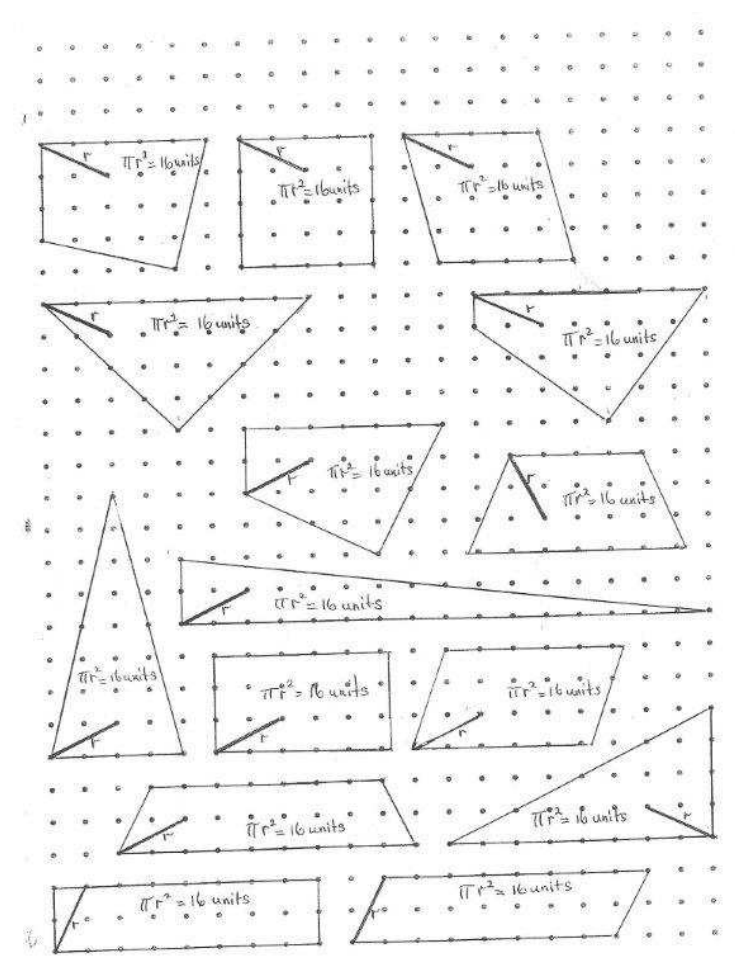

Figure 15

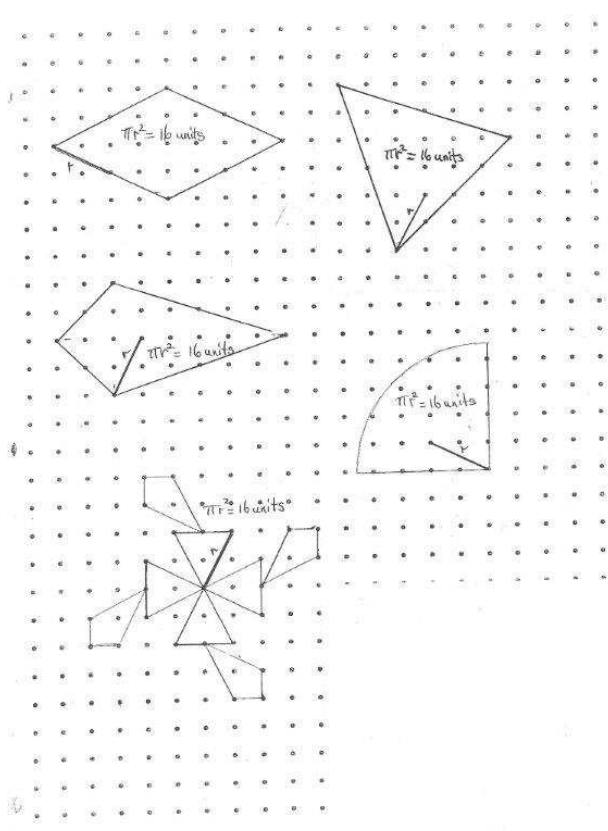

Figure 16

\section{Conclusions}

It is useful to note the following:

- Historical research findings should not necessarily be viewed as the absolute truth, but be subjected to revision;

- Some problems deemed impossible, are so, due to certain ways of thinking, which may be restrictive, but become possible with other ways of visual, creative and critical thinking; 
- Concepts from one area of learning can be applied to other areas to good effect as is evidenced in this paper's original description and definition of a square;

- $\pi$ is a feature of Euclidean Geometry, this is a mystery, but not of the kind presented historically; it has implications for the design of matter;

- $\pi$ is rational, it has an exact value of 3.2 .

- Approximate solutions are just that. However improved, there is no one-to-one correspondence of approximate and exact. Exact means finite and known.

- Existing formulae featuring $\pi$ are unchanged qualitatively, but changes slightly, quantitatively.

The area of any shape has always been defined in terms of the area of an arbitrary unit square. There has never been an issue over the area of any square. It was different for a circle since its area was defined in terms of $\pi$. The unique connection between a square and circle of equal area based on an original parameter, designated secondary median relative to the square, and radius relative to the circle, having the same size changed this, allowing the exact calculation of $\pi$.

* Dotted grid use allows elimination of arcs of construction, hence clean diagrams.

* Each dot is the vertex of the square.

\section{References}

[1] Tapson, Frank, "Oxford Mathematics Study Dictionary", p.46, Oxford University Press, 2006
[2] Biderman, Arthur, "Lexicon Universal Encyclopedia", pp.287, Lexicon Publications Inc. New York, NY, USA, 1989

[3] King, Robert H, "The World Book Encyclopedia" Volume 4, P. 434, World Book Inc., 1982

[4] Orfan, Lucy J, Vogeli, Bruce R, "Mathematics", pp.262-263, 334-335, 342-343,Silver, Burdett and Ginn Inc., 1987

[5] The definition of Pi. (2014, February 6). Retrieved from http://en.wikipedia.org/wiki/Pi

[6] Understanding Pi and Squaring the Circle. (2014, February 6). Retrieved from http://www.docstoc.com/docs/117937307/Understanding-Piand-Squaring-the-Circle

[7] History of Pi and Squaring the Circle. (2014, February 6). Retrieved from http://www-history.mes.st-and.ac.uk/HistTopics/Squaring_th e_circle.html

[8] Layne, C.E, Bostock, L, Shephard, A, Ali FW, Chandler, S, Smith, E, "STP Caribbean Mathematics for CXC Book 4" p.226, Nelson Thomas Ltd. UK, 2005

[9] Goldberg, Nicholas, Cameron- Edwards, Nova, "Oxford Mathematics for the Caribbean", p. 41, Oxford University Press, UK

[10] Bremigan, Elizabeth G, Mathematics for Secondary School Teachers ISBN 978-0-88385-773-1, Mathematical Association of America (n.d). 